\title{
Simbología del poder. El caso de las puertas en las unidades de hospitalización psiquiátrica
}

Symbols of Power. The Case of Doors in Acute Psychiatric Wards

\author{
Carlos Aguilera Serrano', Carmen Heredia Pareja
}

${ }^{1} \mathrm{PhD}$. Enfermero Especialista en Salud Mental. Antropólogo. USMC Motril, Área de Gestión Sanitaria Sur de Granada. Motril, España. ${ }^{2}$ Terapeuta Ocupacional. Antropóloga. USMI-J, Hospital Regional Universitario de Málaga. Málaga, España

Contacto: c_aguiler2@hotmail.com

Fecha de recepción: 20 de diciembre de 2018 / Fecha de aceptación: 12 de septiembre de 2019

\begin{abstract}
Resumen
¿Por qué en algunas unidades de hospitalización psiquiátrica existe hasta una doble puerta de acceso? ¿Qué explicación se da a la existencia de puertas acorazadas? ¿Se guarda o esconde "algo" que la sociedad necesita invisibilizar? ¿Alguien ha medido los efectos terapéuticos del diseño, espacio y estructura arquitectónica de las unidades de hospitalización psiquiátrica?

El medio y sus elementos, incluyendo el conjunto formado por el espacio del servicio, el mobiliario y el material que utilizan los pacientes durante su estancia en un centro hospitalario, influyen en los resultados de salud. En este trabajo de aporte teórico, se analiza el elemento aparentemente formal que configura un objeto artístico, la puerta, y que sin embargo es, en cuanto portador de valores simbólicos, referente de un corpus ideológico contextualizado y producido en las unidades de hospitalización psiquiátrica.

El valor simbólico de la puerta facilita, a la vez que permite, la comprensión de procesos o fenómenos inherentes a las prácticas, atención y cuidados prestados hacia y con las personas ingresadas, pudiendo llegar a conformarse el área privada e íntima que constituye la unidad de hospitalización psiquiátrica en un espacio opresivo y hostil hacia la persona.
\end{abstract}

Palabras clave: planificación ambiental, simbolismo, servicios de salud, hospitalización.

\begin{abstract}
Why in some acute psychiatric wards there is even a double access door? What explanation do we give to the existence of armored doors? Do we save or hide "something" that society needs to make invisible? Has anyone measured the therapeutic effects of the design, space and architectural structure of acute psychiatric wards?

The environment and its elements, including the set formed by the space of the service, the furniture and the material that the patients use during their stay in a hospital center, influence the health results. In this work of theoretical contribution, we analyze the apparently formal element that forms an artistic object, the door, and that nevertheless is, as a bearer of symbolic values, a referent of an ideological corpus contextualized and produced in acute psychiatric wards.

The symbolic value of the door facilitates, at the same time it allows, the understanding of processes or phenomena inherent to the practices, attention and care provided to and with the people admitted, and the private and intimate area that constitutes the acute psychiatric wards in an oppressive and hostile space towards the person.
\end{abstract}

Keywords: environment designs, symbolism, health services, hospitalization.

\section{Contextualización}

En este trabajo de aporte teórico se hace referencia al elemento arquitectónico aparentemente formal que configura un objeto privativo, la puerta, y que sin embargo es, en cuanto portador de valores simbólicos, referente de un corpus ideológico que es el que lo ha producido. Según el diccionario de la Real Academia de la Lengua Española, la significación del término puerta se corresponde con: "Armazón de madera, 
hierro u otra materia que, engoznada o puesta en el quicio y asegurada por el otro lado con llave, cerrojo u otro instrumento, sirve para impedir la entrada y salida, para cerrar o abrir un armario o un mueble"". Otra válida significación la constituye, desde el plano simbólico, aquella que posiciona a la puerta como un símbolo universal que implica transición, dando paso de un dominio a otro; lugar de paso entre dos estados completamente distintos, en donde lo conocido pasa a ser desconocido, convirtiéndose en la abertura que nos permite entrar y salir, cambiar de nivel, de medio, de centro, de vida. Es un transitar hacia otro lugar ${ }^{2}$.

Importantes filósofos, antropólogos, historiadores, escritores, así como arquitectos, diseñadores y otras ramas o disciplinas han contribuido a la elaboración del amplio abanico de connotaciones que incluye la puerta como objeto ${ }^{3-4}$. Secundando al mismo, se ahíncan el objetivo de teorizar sobre el valor simbólico de la puerta en un contexto concreto como son las unidades de hospitalización psiquiátrica. La importancia que dicho elemento adquiere sobre todos los implicados en dicho espacio (profesionales, usuarias/os y/o familiares) así como sobre al ambiente, dinámica y funcionamiento, le confiere gran interés para su análisis. Previamente, se hace necesario especificar el setting, las unidades de hospitalización psiquiátrica, aglutinando bajo esta denominación a todas aquellas unidades y/o dispositivos especializados para la atención a las necesidades de hospitalización en salud mental de la población, las cuales prestan, por lo general, una atención especializada en régimen de hospitalización completa y de corta estancia con intervención intensiva orientada hacia la posterior continuidad de cuidados en la comunidad.

Estudios etnográficos sobre la vida cotidiana de la hospitalización psiquiátrica que abordan la importancia de los espacios, estructuras y elementos configurativos de las instituciones dedicadas a la atención de los problemas de salud mental han sido publicados en el tiempo, aunque quizás el de mayor repercusión mediática, y pese al transcurrir de los años, sean los ensayos realizados por Erving Goffman a mediados del s. XX sobre la situación social de los enfermos mentales ${ }^{5}$. Por entonces, Goffman ya establecía algunas pinceladas acerca de cómo el medio y sus elementos, incluyendo el conjunto formado por el espacio del servicio, el mobiliario y el material que utilizaba el paciente durante su estancia en el centro hospitalario, influían en los resultados de salud de los pacientes. En consonancia con estos datos, en el estudio de Pacheco sobre
La construcción social del enfermo mental, también se hace referencia a este tipo de elementos, que aunque no son determinantes, sí que condicionan la acción de los profesionales sanitarios, y en este caso en concreto, en el quehacer del cuidado enfermero con el paciente y familia ${ }^{6}$.

\section{Análisis en la práctica sanitaria}

Inherente a la definición de espacio, entorno, territorio y/o lugar está implícita la connotación de separación, lo que implica la existencia de un elemento que define un límite, que en este caso se correspondería con la puerta. Lo acotado refiere pertinentemente una barrera impuesta que diferencia y organiza. Así, la puerta de una unidad de hospitalización psiquiátrica se perfila como un elemento que incide en la configuración del espacio caracterizándolo en cuanto a forma, uso y percepción. El valor simbólico de la puerta facilita, a la vez que permite, la comprensión de procesos o fenómenos inherentes a las prácticas, atención y cuidados prestados hacia y con las personas ingresadas, pudiendo llegar a conformarse el área privada e íntima que constituye la unidad de hospitalización en un espacio opresivo y hostil hacia la persona. La división que la puerta hace del espacio público (el exterior; la calle) del espacio privado e íntimo que constituye la unidad se podría interpretar como una manifestación física de la aún permanente subordinación de las/os usuarias/os de salud mental al sistema y, en definitiva, de una sociedad aún estigmatizadora, donde los estereotipos y prejuicios hacia las personas con enfermedad mental influyen en el self construido social e intersubjetivamente.

Dependiendo de la persona implicada, la puerta puede tener un valor o significado distinto. Así, algunas/os usuarias/os pueden percibirla como un elemento de protección, al facilitarles esa separación de la sociedad que les permite y facilita el aislamiento en el espacio configurado por la propia unidad de hospitalización; para otras/os usuarias/os, la puerta puede suponer un elemento que les priva de libertad, al sentirse recluidos y sin tener en cuenta su voluntad, en un espacio cerrado destinado a tal efecto. Para las/os profesionales, podría considerarse como un elemento de seguridad en dos vertientes: por un lado, al facilitar la vigilancia y proximidad con las/os usuarias/os; por otro, la puerta puede suponer la clave en el modo de percibir el control del entorno, donde el/la paciente, como ya describía Goffman", sufriría una "Desculturación" ("desentrenamiento que lo incapacita temporalmente 
para encarar ciertos aspectos de la vida diaria en el exterior"), estableciéndose así unas relaciones interpersonales desiguales y distantes de lo terapéutico. El estudio etnográfico sobre la estructura social de los hospitales psiquiátricos de Strauss et al., en 1964, muestra y defiende también las articulaciones entre las ideologías que guían las prácticas de los profesionales y las formas en que los pacientes interpretan sus padecimientos $^{7}$ : “... el orden institucional no constituye una fuerza exterior que controla a los individuos pasivamente, sino que los mismos pacientes participan en su construcción".

Estas son algunas interpretaciones sobre el alcance simbólico de las puertas en las unidades de hospitalización psiquiátrica, siendo varios los interrogantes acerca de su funcionalidad y valor en la actualidad: ¿Por qué en algunas unidades de hospitalización psiquiátrica existe hasta una doble puerta en el acceso a la misma? ¿Qué explicación damos a la existencia de puertas acorazadas? ¿Qué es lo que preocupa o asusta tanto para necesitar una puerta "cerrada a cal y canto"? ¿Nos asusta lo de afuera, para evitar que entre?, o acaso ¿se guarda o esconde "algo" que la sociedad necesita invisibilizar? ¿Alguien ha medido los efectos terapéuticos del diseño, espacio y estructura arquitectónica de las unidades de hospitalización psiquiátrica?

Algunos de estos cuestionamientos quizás no tengan fácil ni rápida respuesta por su ambigüedad e, incluso, por la falta de uniformidad en relación a las políticas, normas, principios y reglas que guían las prácticas y modos de actuación en estos servicios de atención sanitaria. Sin embargo, experiencias y estudios recientes en Alemania, Reino Unido, Italia o incluso España, lamentablemente aún excepcionales, indican que la política de puertas cerradas en los hospitales de salud mental no es mejor que las de puertas abiertas, por ejemplo, en la prevención del suicidio o frente a las conductas de fuga ${ }^{8-10}$. En una revisión bibliográfica de artículos empíricos en 2009 sobre puertas cerradas en salas de internación psiquiátrica aguda, se indica que las salas bloqueadas se asociaron con una mayor agresión del paciente, una menor satisfacción con el tratamiento y una exacerbación de los síntomas ${ }^{11}$. Además, se les asocia también a las unidades de puertas abiertas con una disminución significativa de las medidas coercitivas, lo que viene a contribuir y facilitar un buen modelo de atención, fortaleciendo y salvaguardando el derecho de autonomía de los/as usuarios/as y facilitando su autodeterminación ${ }^{12}$. Así, se ha comprobado como la apertura de salas psi- quiátricas cerradas puede ayudar a establecer un ambiente terapéutico positivo, sin omitir o poner en riesgo los cuidados prestados hacia usuarias/os con requerimientos y/o cuidados más exhaustivos por la gravedad de su situación clínica ${ }^{13}$, y, en definitiva, promoviendo mejores resultados en la salud de las personas hospitalizadas ${ }^{9}$.

\section{Conclusiones}

Las instituciones, junto con los profesionales y los grupos de población implicados, deben aunar esfuerzos para trabajar en el rediseño y remodelado de los espacios utilizados en la atención a la salud mental, intentando hacerlos más amables, cercanos, accesibles. La meta no es otra que la de contribuir a la eliminación de todas aquellas estructuras, elementos, iconos o signos que transmitan y/o reproduzcan poder, dominación, desigualdad, discriminación, y en definitiva, toda forma de violencia simbólica que termina por naturalizar el estigma, la subordinación y el rechazo social de las personas con problemas de salud mental.

La naturaleza compleja en el caso de la puerta como elemento físico de las unidades de hospitalización psiquiátrica, pero, también, como elemento de importante carga simbólica y social, merece una investigación más a fondo y exhaustiva que facilite cuantificar la repercusión en los resultados de salud así como en el bienestar y satisfacción de los sujetos.

El futuro del cuidado profesionalizado y tratamiento en salud mental, incluyendo la forma, el modo, el espacio y en definitiva, el contexto y las condiciones, pasa por desarrollar prácticas basadas en los principios de la ética, garantizando el respeto a la dignidad y autonomía personal, y trabajando para ello con una política de defensa del ambiente terapéutico que facilite a las personas gozar de un entorno saludable, apropiado, seguro, privado y digno.

\section{Bibliografía}

1. Varela Iglesias J, Tadeo Monge F, Carreño Rujillo G, Doménech Colón F, Abad León A, Reta Janariz A, et al. Real Academia Española. Diccionario Usual. Edición del Tricentenario. 2014. p. 1.

2. McClue J. El símbolo de la puerta y sus significados. [Internet]. Simbolos, mitos y arquetipos. 2016. Available from: http://abajocomoarriba.blogspot.com/2016/11/el-simbolo-dela-puerta-y-sus.html

3. Sartre JP. A Puerta Cerrada. Buenos Aires: Editorial Losada. S.A.; 1960.

4. Sánchez Pérez F. El espacio y sus símbolos: Antropología de la casa Andaluza. Reis Rev española Investig sociológicas. 1990;52(90):47-64. 
5. Goffman E. Internados. Ensayos sobre la situación social de los enfermos mentales. 3a ed. Buenos Aires: Amorrortu; 2001.

6. Pacheco Borrella G. La construcción social enfermo mental. Saarbrücken (Alemania): Academia Española; 2013. 1-559 p.

7. Strauss A, Schatzman L, Bucher R, Ehrlich D, Sabshin M, Al. E. Psychiatric ideologies and institutions. Londres: CollinMacMillan; 1964.

8. Mezzina R. Community mental health care in Trieste and beyond: an "open door-no restraint" system of care for recovery and citizenship. J Nerv Ment Dis [Internet]. 2014;202(6):440-5. Available from: http://www.ncbi.nlm.nih.gov/pubmed/24840089

9. Huber CG, Schneeberger AR, Kowalinski E, Fröhlich D, von Felten S, Walter $\mathrm{M}$, et al. Suicide risk and absconding in psychiatric hospitals with and without open door policies: a 15 year, observational study. The Lancet Psychiatry. 2016;3(9):842-9.

10. Amorós M. Psiquiatría sin llaves ni cerrojos. El Mundo. Palma de Mallorca; 2016 Mar 21:

11. Van Der Merwe M, Bowers L, Jones J, Simpson A, Haglund K. Locked doors in acute inpatient psychiatry: A literature review. J Psychiatr Ment Health Nurs. 2009;16(3):293-9.

12. Jungfer HA, Schneeberger AR, Borgwardt $S$, Walter $M$, Vogel $M$ Gairing SK, et al. Reduction of seclusion on a hospital-wide level: Successful implementation of a less restrictive policy. J Psychiatr Res. 2014;54(1):94-9.

13. Blaesi S, Gairing SK, Walter M, Lang UE, Huber CG. [Safety, therapeutic hold, and patient's cohesion on closed, recently opened, and open psychiatric wards]. Psychiatr Prax [Internet]. 2015;42(2):76-81. Available from: https://www.thiemeconnect.com/DOI/DOI?10.1055/s-0033-1359871 